\title{
Particle Physics Masterclasses: Sharing LHC Research and Discovery with High School Students
}

\author{
Uta Bilow ${ }^{1}$ \\ Technische Universität Dresden \\ Institute of Nuclear and Particle Physics, TU Dresden, 01062 Dresden, Germany \\ E-mail: uta.bilowltu-dresden.de
}

\begin{abstract}
Masterclasses are a proven tool to engage high school students with particle physics. In a Masterclass, participants learn about high energy physics in a day-long course. Moreover, they can actively take part in cutting-edge research and experience methods and tools used in research by performing a tailor-made physics analysis involving real LHC data under the supervision of physicists. During a Masterclass students not only explore the fundamental forces and building blocks of nature, but they can also improve their understanding of science and the scientific discovery process. Particle Physics Masterclasses offer an authentic experience and add a new dimension to physics education at school, thus stimulating the students' interest in science. More than 300 Masterclasses are organized in the framework of the program International Masterclasses each year. During a 6-week period spanning roughly February to April, research institutes and universities in more than 50 countries invite high school students for a Masterclass. At the end of each day, the participants join in a videoconference with other student groups and CERN, Fermilab, or TRIUMF. Like an international research collaboration, they share and combine the results of their analyses and can thus experience for themselves an important part of the experimental particle physics working process. The program attracts more than 13,000 high school students each year and is run by IPPOG, the International Particle Physics Outreach Group, and is centrally coordinated at Technische Universität Dresden and University of Notre Dame. The successful Particle Physics Masterclasses are also arranged in other contexts. In 2017, an extra effort was started by IPPOG to support and promote the access of girls to science. To accomplish this, IPPOG launched Masterclass activities especially for girls on the UN International Day of Women and Girls in Science. In Germany, the nationwide program Netzwerk Teilchenwelt, which includes 30 universities and research labs, has been established. For Netzwerk Teilchenwelt, Particle Physics Masterclasses are instrumental as a basic level, but is builds up several higher levels of engagement, e.g. workshops at CERN or research projects at universities and institutes for the most motivated and interested high school students. On total, this program offers activities for 4000 high school students per year on four levels. In the United States, QuarkNet offers International Masterclasses, a World Wide Data Day with LHC data, and Masterclass preparation activities as important components of a program to bring particle physics to high school teachers and students.
\end{abstract}

The 39th International Conference on High Energy Physics (ICHEP2018)

4-11 July, 2018

Seoul, Korea

${ }^{1}$ Speaker 


\section{Introduction}

Particle physics is attracting great public interest. The field deals with fundamental questions such as the origin of the universe and the structure of matter at the most basic level. In addition, its vocabulary is enriched with fascinating terms such as the Big Bang and antimatter. And scientists use extraordinary experimental technology such as the Large Hadron Collider (LHC) and the detectors that gather vast amounts of data from it. These special features of High Energy Physics (HEP), together with important and exciting discoveries, have led to a strong presence of particle physics in the media. However, the subject is rarely addressed in school and in science curricula, so it is too often difficult if not impossible to teach this cutting-edge physics to high school students. Particle Physics Masterclasses close this gap. They offer high school students the opportunity to learn about HEP in a one-day course with help of physicists. In this way, young people can be informed about findings, research questions and discoveries. Masterclasses also serve to inspire the next generation of physicists and they help to create an appreciation for basic science.

\section{The Concept of Masterclasses}

The format of a "Masterclass" is most often found in the arts, where a student works with an expert artist, such as a painter or a violinist. The concept is adaptable to particle physics if the expert is a physicist and the subject is data analysis. In a Particle Physics Masterclass, high school students follow in the footsteps of a scientist and not only listen to lectures but do a hands-on activity, using the same methods and tool as the scientist. LHC Masterclasses that meet these requirements are organized in several programs. The most prominent example is the International Masterclasses (IMC) [1]. The program is organized by the International Particle Physics Outreach Group (IPPOG), a network of scientists, educators and communication specialists working in informal science education and outreach for particle physics. In Germany, Masterclasses are offered nationwide via the national project Netzwerk Teilchenwelt. In addition, there are many more applications in different settings in a large number of countries. A typical Masterclass consists of introductory presentations, a tour of physics facilities, a tailormade physics analysis with LHC data, and a videoconference with international participation and connection to CERN or Fermilab. International Masterclasses are organized every year in spring and coordinated at Technische Universität Dresden (Germany) and QuarkNet (U.S.). Since its launch in 2005, the program has grown steadily. Meanwhile 52 countries are involved, 225 universities or research labs participate, and reach thereby approximately 14.000 high school students per year.

\subsection{Measurements with LHC data}

The four large LHC experiments have designed six different measurement packages and made available data for Masterclasses. The packages have been translated in up to 21 languages to lower the barrier for young students. Two of these measurements are briefly outlined here: In the so-called ATLAS Z path [2], students look at event displays and identify events with 2 or 4 leptons or di-photon events. A tool calculates the invariant mass, and mass histograms are 
created. This way, students can measure the mass of known particles such as the Z, Upsilon, J/Psi, and the Higgs. MC events have been mixed into the data sample, so students can also "discover" a Z' with a mass of $\sim 1000 \mathrm{GeV}$. Each pair of students analyzes 50 events, and all results are combined, so that a group of 20 students has sufficient statistics to look into the concepts of statistics, modeling, and signal significance. When students discover the various distributions of particle masses, they get insights into the process of discovery and how physicists understand data. The package with CMS data [3], called the WZH measurement, provides students with a three-dimensional online event display using WebGL. Students characterize W, Z, and Higgs candidate events and create mass plots of particles that decay into 2 leptons. In addition, they determine the ratios of electrons to muons and of $\mathrm{W}+$ to $\mathrm{W}$ particles, which gives them insight into the structure of the proton.

\subsection{Masterclasses for Girls}

Since 2017, LHC Masterclasses are held at the International Day of Women and Girls in Science (IDWGS). In 2015, the United Nations General Assembly established February 11 as the International Day of Women and Girls in Science. This date should be recognized as a global celebration of the equal participation and accomplishments of female scientists. Awarenessraising events are encouraged on this date to promote the access of women and girls to science education and research activities. IPPOG followed this call by organizing a special edition of Masterclasses on the IDWGS. In order to follow the idea of the IDWGS, institutes were encouraged to arrange Masterclasses for girls only and to involve as many female scientists as possible as tutors. The videoconferences at CERN are run by female moderators and the program is enriched with a presentation on the situation of women in physics.

\section{Netzwerk Teilchenwelt}

LHC Masterclasses are typically one-day activities. Several initiatives have established more sustainable programs, combining the Masterclass event with a follow-up. A very prominent example is the Germany-wide Netzwerk Teilchenwelt (NTW). This network is formed by 30 universities in Germany in close contact with CERN, and the program is led at Technische Universität Dresden. NTW comprises a multi-level program for about 4000 high school students per year. On the basic level, around 140 Masterclasses are held throughout the year at schools, museums, or science centers. Advanced students can work with detectors on astroparticle physics projects or analyze data from the web portal Cosmic@,Web. The program goes well beyond Masterclasses. The most motivated and committed students -60 per year are invited to visit CERN for a 4-days-workshop. At the top level, students can immerse themselves even deeper in HEP. They are invited to CERN for 2 weeks and carry out their own research projects. These projects will be prepared in advance and subsequently supervised at one of NTW's 30 universities and labs.

In order to promote and maintain young people's commitment to particle physics, a new part of the project, the so-called Fellow Program, was launched in 2017. This program tightens the link between these already trained and motivated students and the research groups at the universities and creates mutual benefit. 120 Fellows have subscribed so far and take advantage of the program: They get close connections to the research groups and are offered internships, 
mentoring, and invitations to special events. On the other hand, the research labs benefit from highly motivated students as future members of the groups who support scientific activities and outreach activities.

\section{QuarkNet as a Partner}

QuarkNet, the U.S. based teacher network, is a strong partner in Masterclasses and related activities. QuarkNet coordinates International Masterclasses for those countries that connect to a videoconference with Fermilab due to their time zone, and organizes preparation activities for participants. QuarkNet is also responsible for the development and maintenance of the CMS Masterclass. Most recently, QuarkNet launched World Wide Data Day, a related annual activity, where students can analyze LHC data with a more "simple" measurement that is usually carried out at school under supervision of a physics teacher and does not necessarily need the direct involvement of particle physicists, in contrast to a Particle Physics Masterclass.

\section{How to get involved}

Research labs and Particle Physicists are strongly encouraged to contribute to the program and help to inspire the next generation of physicists. Institutes and physicists interested in joining the International Masterclasses program and organizing an event at their research lab should contact the coordinators. Physicists based at CERN or Fermilab can support the program by moderating videoconferences with high school students. All of the material for Particle Physics Masterclasses is available on the web and free to use for any educational purpose.

\section{Outlook}

Particle Physics Masterclasses are an established means of bringing high school students into contact with particle physics. Studies have shown that Masterclasses promote young people's interest in modern physics [5]. This successful concept has been adapted by other communities: IceCube Masterclasses have run since 2014, and neutrino Masterclasses with MINERvA data will be launched in 2019. Further experiments are currently developing measurements for high school students, e.g. Belle II.

\section{References}

[1] M. Bardeen, H.P. Beck, U. Bilow, K. Cecire, F. Ould-Saada, M. Kobel, International Masterclasses in the LHC era, CERN Courier May 22, 2014, http://cerncourier.com/cws/article/cern/57305.

[2] E. Gramstad, The challenge of explaining new physics concepts and phenomena, PoS (EPSHEP2017) 556.

[3] K. Cecire, T. McCauley, The QuarkNet CMS masterclass: bringing the LHC to students, Nucl. Part. Phys. Proc., 273-275, 1261.

[4] U. Bilow, International Masterclasses in Particle Physics - An excellent instrument to inspire highschool students, PoS (EPS-HEP2017) 818.

[5] K.E. Johansson, M. Kobel, D. Hillebrandt, K. Engeln, M. Euler, European particle physics masterclasses make students into scientists for a day, Phys. Educ., 42 (6), 636. 